corresponding family have in common a finite number $\nu^{n}$ of points (or $n-1$-flats). And all loci (or envelopes) of order (or class) $\nu$ having in common $C-n+1$ fixed points (or $n-1$-flats) have also in common $\nu^{n}-C+n-1$ other fixed points (or $\overline{n-1}$-flats).

The maximum number $C$ of determining elements is unchanged by the exchange of $n$ and $\nu$, but such is not in general true of the minimum number of extra determined elements; for the only positive integral solutions of the equation, $\nu^{n}-C+n-1=n^{\nu}-C+\nu-1$, are: (1) $\nu$ or $n$ $=1, n$ or $\nu=1,2,3, \cdots ;(2) \nu$ or $n=2, n$ or $\nu=3$. Case (2) yields interesting familiar theorems in 2- and 3-fold space. Excepting cases (1) and (2) there subsists the inequality, $\nu^{n}-C+n-1 \neq n^{\nu}-C+\nu-1$, where the right or the left member is the greater according as $\nu>n$ or $n>\nu$. When $m$ is less than both $n$ and $\nu$, there is a corresponding inequality whose sense has the same criterion as above, though now the number of additional determined elements is infinite.

Columbia University.

F. N. Cole.

\title{
THE EVANSTON MEETING OF THE CHICAGO SECTION.
}

THE second meeting of the Chicago Section of the American Mathematical Society was held at Northwestern University, Evanston, on Thursday and Friday, December 30 and 31, 1897, Professor E. H. Moore, Vice-President of the Society, presiding.

The following members of the Society were present: Professor Henry Benner, Dr. E. M. Blake, Professor Oskar Bolza, Mr. A. C. Burnham, Professor Ellery W. Davis, Dr. L. W. Dowling, Dr. James W. Glover, Professor Arthur S. Hathaway, Professor Thomas F. Holgate, Mr. H. G. Keppel, Professor Joseph L. Markley, Professor Heinrich Maschke, Professor Malcolm McNeil, Professor E. H. Moore, Professor H. B. Newson, Professor J. B. Shaw, Dr. H. F. Stecker, Professor C. A. Waldo, Professor Henry S. White, Professor Mary F. Winston, Professor Alexander Ziwet.

The two days' session was fully occupied in reading the following papers : 
1. Professor Heinrich Maschke: "The determination of all ternary and quaternary symmetrical and alternating collineation groups."

2. Dr. G. A. Miller : "On the solvability of groups."

3. Professor H. B. Newson : "Normal forms of projective transformations" (preliminary communication).

4. Professors Newson and Hathaway: "Continuous groups of spherical transformations" (preliminary communication).

5. Professor Oskar Bolza: "On the cubic involution and the theory of elliptic functions."

6. Dr. James W. Glover : "A generalized Legendre's coefficient" (preliminary communication).

7. Professor Arthur S. Hathaway: "Alternate processes."

8. Professor Henry S. White : "Twisted quartic curves of the first species and certain associated quartics."

9. Professor James Byrnie Shaw : "Report on Cantor's transfinite numbers.'"

10. Mr. Edgar J. Goodspeed : "On a geometrical papyrus of the first century."

11. Dr. James H. Boyd (introduced by Professor Moore): "Approximate solution of a particular differential equation."

12. Professor Henry Benner : "Independent computation of integrals involving the square root of a quadric or cubic expression."

13. Professor E. H. Moone: "Concerning the general equations of the seventh and eighth degrees."

14. Dr. L. W. Dowling: "On systems of curves dependent on a single parameter" (preliminary communication).

15. Dr. E. M. Blake : "On a ruled surface of the fourth order mechanically generated."

16. Dr. E. M. BLAKe : "On articulated systems", (second preliminary communication).

17. Mr. Carl C. Engberg (presented by Professor E. W. Davis): "Note on the triple focus of the Cartesian Oval."

18. Mr. D. N. Lehmer (presented by Professor E. W. Davis): "Multiple totients."

19. Professor Ellery W. Davis: "A note on reticulations."'

20. Dr. L. E. Dickson : "Decomposition of the orthogonal group."

21. Professor C. H. Hinton : "On a remarkable class of hyperspherical tetrahedra" (preliminary communication). 
In Professor Maschke's paper (No. 1), which is to be published in the Mathematische Annalen, a collineation group which is holohedrically isomorphic with the symmetric or alternating substitution group on $k$ letters $G_{k !}$ or $G_{1 / 6 !}$ is extended to a group holohedrically isomorphic with a $G_{(k+1) !}$ or $G_{3 / 3(k+1) !}$ by the determination of an additional collineation. Starting from the lowest values of $k$ for which the corresponding collineation groups can easily be obtained the method leads by this extension to all possible groups of the required property, considering all those groups as identical that can be linearly transformed into each other.

Abstract of Dr. Miller's paper (No. 2): A group is said to be solvable when its ath derivative is unity. If a group is insolvable its $\alpha$ th derivative is perfect and its order exceeds unity. This perfect group includes all the composite factors of composition of the original group. Hence the study of insolvable groups is reduced to that of perfect groups. Every perfect group has an $\alpha, 1$, isomorphism to a simple group of composite order. If it is represented as an intransitive substitution group all its transitive constituents must be perfect. Any simple quotient group of a compound group of degree $n$ may be represented as a transitive group whose degree is less than $n$. The lowest order of a compound perfect group is 120 and the lowest degree of such a group, when it is represented as a substitution group, is 8 . This paper will probably be published in the American Journal of Mathematics under the more special title "On the perfect groups."

Professor Newson's paper (No. 3) will be published, when complete, in the Kansas University Quarterly.

Professor Bolza's paper (No. 5) will be published in full in the Mathematische Annalen. If the values of the variable $X=X_{1} ; \quad X_{2}$ of a cubic involution are represented by the points of a conic, the triangles whose vertices represent the triples of the involution are circumscribed to a fixed conic, Weyr's involution conic. The theory of Poncelet polygons $(n=3)$ furnishes then a connection with the theory of elliptic functions. In this paper the connection thus established between the theory of cubic involutions and the theory of elliptic functions is investigated in detail.

Abstract of Professor Hathaway's paper (No. 7): The alternate process $A_{(s)}$ is equivalent to $\frac{1}{m} \sum_{r=1}^{r=m} \pm S_{r}$, where $\pm S_{r}$ is the substitution $S_{r}$ with the factor +1 or -1 according as it is an even or an odd number of transpositions. For the 
symmetric process $C_{(s)}$, the factor \pm 1 is replaced by +1 . There is a duality between "alternate" and "symmetric," and also between "place" and "variable" substitutions. The principal theorem is $A_{(t)}=A_{(s)} A_{\left(s^{\prime}\right)}$ where $(t)=(s)\left(s^{\prime}\right)$. The processes are illustrated by applications to determinants, quaternions, and four-fold and $n$-fold space.

Professor White's paper (No. 8) may be summarized as follows : To every quadric surface $F_{2}$ containing the curve $C^{4}$ there may be assigned an index showing how many generators from each of its two reguli are required to constitute a closed inscribed polygonal line. The sides of such a polygonal line may be rearranged in a manner analogous to the sides of a star-polygon giving rise to new vertices, The locus of new vertices is, for each rearrangement a new $C^{4}$ on the $F_{2}$, covariantly associated with the former $C^{4}$. Variation of the $F_{2}$ varies the new $C^{4}$, generating a new covariant locus, whose nature can be investigated by the aid of plane projections and of known formulæ due to Salmon.

Mr. Goodspeed's paper "On a geometrical papyrus of the first century" was a report of his investigations on a papyrus purchased in Cairo by Mr. E. E. Ayer, of Chicago, now lying in the Egyptian room of the Field Columbian Museum, and believed to be a copy of work by Hero of Alexandria. The report, which was made at the request of the programme committee, dealt with the internal evidences of the papyrus which serve to locate it and embraced a translation of the writing.

Dr. Boyd's paper (No. 11) presented a practical method for finding the approximate general integral of the equation

$$
\frac{d y}{d x}=X_{0}+X_{1} y+X_{2} y^{2}
$$

where $X_{0}, X_{1}, X_{2}$ are any functions of $x$.

The problem set was to give a method for finding a function of $x$ which, substituted for $y$ in the differential equation, would leave a remainder which could be made as small as desired by repeating the processes of the method often enough.

Professor Benner's paper (No. 12) gave a method for the independent determination of the coefficients which appear in the computation of the integrals

$$
\int \frac{x^{n} d x}{\sqrt{1+a x+b x^{2}}} \text { and } \int \frac{x^{n} d x}{\sqrt{1+a x+b x^{2}+c x^{3}}}
$$


Professor Moore's paper (No. 13) treats of the interrelations of the equations mentioned in the title after adjunction of the square roots of their discriminants and their total resolvents of degree 15 , dependent upon the triple system in 7 letters and the quadruple system in 8 letters. The paper will be offered to the Mathematische Annalen for publication.

Dr. Blake's paper (No. 15) is a continuation of his investigations upon mechanisms which generate ruled surfaces.

If a plane $m_{1}$ containing two fixed points $p_{1}$ and $p_{2}$ slides upon an incident plane $m_{2}$ containing two fixed straight lines $l_{1}$ and $l_{2}$ in such a manner that $p_{1}$ always remains upon $l_{1}$, and $p_{2}$ upon $l_{2}$, then any point of $m_{1}$ traces an ellipse upon $m_{2}$ and any point of $m_{2}$ traces a limaçon upon $m_{1}$. The paper is devoted to the study of the surface generated by the line rigidly attached to $m_{2}$ and passing through the intersection of $l_{1}$ and $l_{2}$, when $m_{1}$ is regarded as fixed. The surface is of the fourth order and fourth class and its Cartesian equation is $2\left(x^{2}+y^{2}-2 \alpha x\right)^{2}=z^{2}\left(x^{2}+y^{2}\right)$. The paper was illustrated by a thread model.

Mr. Carl C. Engberg (No. 17) showed that in order to satisfy the numbers for the Plückerian characteristics the triple focus of the Cartesian oval should be counted double only.

In Mr. D. N. Lehmer's paper (No. 18) multiple totients are defined thus: The ordinary totient of $k$ is the number of numbers $X$ not greater than $k$ and prime to it; the double totient of $k$ is the number of pairs of numbers

$$
x_{1}, x_{2} \quad\left(x_{1}<x_{2}<k\right)
$$

such that $x_{1}, x_{2}$, and $k$ have no common factor; similarly triple, $\cdots, n$-tuple totients are defined. These are found to enjoy properties similar to the ordinary totients.

Professor Davis (19) showed that if there be a reticulation on a surface of deficiency $p$, with $F$ for number of faces and $V$ for number of vertices, then it does not necessarily follow from $F$ less than $V$ that there can be another reticulation on the surface with same $F$, but with vertices $V_{1}$ less than $F$.

Dr. Dickson (No. 20) showed that the $m$-ary orthogonal group in the Galois field of order $n$, by its decomposition, leads to a new triply-infinite system of simple groups.

The officers of the Section elected for the ensuing year are : Secretary, Professor Thomas F. Holgate; additional members of the Programme Committee, Professor Arthur S. Hathaway, Professor Heinrich Maschke. 
On the evening of the first day of the session the members present dined together at the Avenue House and a very enjoyable hour was spent.

The next meeting of the Section will take place during the spring of the current year, of which due notice will be given.

Thomas F. Holgate, Secretary of the Section.

\section{THE PHILOSOPHY OF HYPERSPACE.}

PRESIDENTIAL ADDRESS DELIVERED BEFORE THE AMERICAN MATHEMATICAL SOCIETY AT ITS FOURTH ANNUAL MEETING, DECEMBER 29, 1897.

BY PROFESSOR SIMON NEWCOMB.

THERE is a region of mathematical thought which might be called the fairyland of geometry. The geometer here disports himself in a way which, to the non-mathematical thinker, suggests the wild flight of an unbridled imagination rather than the sober sequence of mathematical demonstration. Imaginative he certainly does become, if we apply this term to every conception which lies outside of our human experience. Yet the results of the hypotheses introduced into this imaginary universe are traced out with all the rigor of geometric demonstration. It is quite fitting that one who finds the infinity of space in which our universe is situated too narrow for his use should, in his imaginative power, outdo the ordinary writer of fairy tales, when he evokes a universe sufficiently extended for his purposes.

The introduction of what is now very generally called hyperspace, especially space of more than three dimensions, into mathematics has proved a stumbling block to more than one able philosopher. The question whether a fourth dimension may possibly exist, and whether it can be legitimately employed for any mathematical purpose, is one on which clear ideas are not universal. I do not, however, confine the term "hyperspace" to space of more than three dimensions. A hypothesis which is simpler in its fundamental basis, and yet seems absurd enough in itself, is that of what is sometimes, improperly I think, called curved space. This also we may call hyperspace, defining the latter in general as space in which the axioms of the Euclidean geometry are not true and complete. Curved space and space of four or more dimensions are completely dis- 